fischen Krankheitsstoffes, sondern beweisen nur das Vorhandensein eines wunde Hautstellen zur Blaseneruption reizenden Secretes. Es wäre anders, wenn die Form und der Verlauf der entstandenen Impfblasen eine ganz charakteristische wäre oder wenn zugleich auch an nichtgeimpften Stellen Blasen hervorbrächen. Beides hat aber bisher die Beobachtung nicht gezeigt.

Nach der jetzigen Lage unserer Kenntniss halte ich es für wahrscheinlich, dass wir in dem sogenannten Pemphigus acutus neonatorum keine specifische Krankheit vor uns haben, sondern, dass wir der Haut der Neugeborenen die Eigenschaft zuschreiben müssen, auf Reize, welche sie treffen, seien dieselben nun mechanischer, ehemischer oder auch wohl thermischer Art, mit der Eruption von mehr weniger grossen Blasen zu antworten und dass der Inhalt dieser Blasen bisweilen selbst wieder, auf wunde Hautstellen gebracht, diese zu nener örtlicher Blaseneruption reizen kann.

Die Frau S. habe ich mit dem Rathschlag aus der Anstalt entlassen, dass sie ihre Praxis einschränken und ihre Manipulationen bei dem Besorgen Neugeborener ruhiger und sanfter machen solle, Seit einem Monate ist sie nunmehr wieder in der Praxis und hat, wie sie mir mittheilt, Pemphigusfälle, wie früher, seitdem nicht erlebt.

\title{
Aus der gynäkologischen Klinik zu Breslau.
}

\section{Ueber Echinococcusgeschwïlste des Beckens als Geburtshinderniss.}

\author{
Von
}

\section{Dr. Max Wiener,}

Assistent der Poliklinik.

Eine am 6. August 1876 in der hiesigen gynäkologischen Poliklinik beobachtete, durch Echinococcusgeschwälste des Beckens erschwerte Geburt gab Veranlassung, die in der Literatur verzeichneten ähnlichen Beobachtungen zusammenzustellen, um vielleicht einen sicheren Anhalt für die klinische Seite dieser Complication zu erhalten. Die Ausbeute ist jedoch eine sehr spärliche geworden, da nur ein sehr kleiner Theil der in der Literatur verzeichneten Beckenechinococcen bei graviden Frauen gefunden wurde. Es sind sechs, nämlich 
drei der 31 von Davaine in seinem, "Traité des Entozoaires et des maladies vermineuses de l'homme et des "animaux domestiques" angeführten Echinococcen der verschiedenen Beckenorgane, Obs. 162, 163,164 ; letzterer ist sogar zweifelhaft, wenn es auch wahrscheinlich, dass der später gefundene Hydatidentumor als die bei der acht Jahre vorher stattgehabten Geburt beobachtete Beckengeschwulst aufzufassen ist.

Den vierten hierhergehörigen Fall erzählt Puchelt in seiner "Commentatio de tumoribus in pelvi partum impedientibus," Heidelberg 1840 , S. $213, \S 4$; den fünften Birnbaum in der Monatsschrift für Geburtskunde und Franenkrankheiten, 24. B., S. 428; den sechsten Sadler (ibid. 25. Bd, S. 73). Der siebente Fall ist der bei uns beobachtete.

Da diese sieben Beobachtungen verschiedene gemeinschaftliche Gesichtspunkte aufweisen, sollen sie etwas genauer erzählt werden.

1) Davaine, Obs. 162. Dr. Park (Transact. medico-chirurg. Londres 1817) fand bei einer Ipara am Ende der Gravidität die Vagina fast ganz ausgefüllt von einem harten Tumor, der zwischen Scheide und Rectum lag. Finger nur mit Mühhe zwischen Tumor und Schambein bis zum Mutterhalse gebracht. Geburt nach langer und mühsamer Anstrengung spontan: Später zwei Mal Zwillingsgeburten, 1 Mal im vierten Monate, das zweite Mal Ende des siebenden Monates, ohne Zufall. Während dieser Schwangerschaft von Zeit zu Zeit Urinverhaltung. Neue Schwangerschaft. Geburt am normalen Ende. Oeffnung des Mutterhalses vollständig; Blase gesprungen. Die ganze Nacht schmerzhafte Wehen. Kopf stemmt sich fortwährend gegen die obere Partie des Hindernisses, rückt trotz heftiger Wehen nicht vorwärts. Darauf oberflächliche Incision in den Tumor, Erweiterung des Schnittes mit dem Finger. Entleerung von serös-blutiger Fluissigkeit nebst einer Anzahl von Membranstüeken. Die erste Wehe entleerte den übrigen Inhalt vollkommen, die nachfolgenden vollendeten die Geburt bald. Langsame Erholung, starke Eiterung, Fieber, Nierenschmerzen. Heilung nach 8-10 Wochen.

Darauf noch zwei Male schwierige Entbindungen. Der Grund davon lag, nach Park's Vermuthung, in einem gewissen Grade von Schrumpfung, die durch den Vernarbungsprocess nach der Operation herbeigefuihrt wurde. Bei der zweiten Entbindung am Ende des achten Monates lag ein Arm vor. Park hatte grosse Schwierigkeit, die Hand einzuführen and giebt an, dass das Hinderniss sicherlich nicht im Uterinhalse lag. 1859).

2) Obs. 163. Blot (Comptes rendues Soc. de Biolog., Avril

24jähr. Frau. Tumor der recto-vaginalen Scheidewand. Versehluss der Scheide, absolutes Hinderniss für den Durchtritt des Kopfes. Wehen seit drei Tagen. Punction durch die Scheide mit einem gekrïmmten Troicar. Entleerung: der ganzen Flüssigkeit. Nach 20 Minuten Geburt eines lebenden Kindes. Entleerte Flïssigkeit durch- 
scheinend. Kochen und Salpetersäure geben keinen Niederschlag. Mikroskopische Untersuchùng lässt keine Echinocoecusbestandtheile erkennen. S. 287$)$.

3) Obs. 164. Roux (Journ. de méd. de Sédillot 1828, C. III,

38jährige Frau. Vor acht Jahren lange und schwere Entbindung. Der Geburtshelfer erkannte die Ursache des Hindernisses in einem, in der linken Seite der Scheide gelegenen Tumor. Der Tumor wuchs, ohne die nächsten füinf Jahre Zufälle zu machen. Die folgenden drei Jahre Urin- und Stuhlausscheidung erschwert. Es wurde die Anwesenheit eines harten Tumors constatirt, links gelegen, vom Beckenrande nach der grossen Labie sich erstreckend. Scheide nach der rechten Seite gedrängt. Gefühl von Schwere, schmerzhafter Zerrung im Becken u. s. w. Roux glaubte an die Anwesenheit eines festen Tumors und entschloss sich zur Operation. Beim ersten Messerzuge stürzte eine grosse Menge durchscheinender Flüssigkeit hervor. Mit dem Finger gelangte man in einen weiten Sack, dessen Wünden membranöse Flocken anzuhängen schienen. Mit der Polypenzange zog man eine Membran heraus, die als Hydatide erkannt wurde. Eiterung, Heilung.

4) Puchelt (1. c.).

31jährige Frau. Bei der ersten Geburt wurde eine Geschwulst constatirt, die von der Steissbeinspitze bis über das Promontorium und bis an beide Hüftbeine zu verfolgen und im ganzen Umfange mit diesen Theilen verwachsen war. Nur mit zwei Fingern konnte das iiber den Schambeinen liegende Orificium externum erreicht werden. Der unter den Wehen in die Scheide herabgepresste Tumor war hart, wich dem Drucke des Fingers aus, so dass er deutliche Fluctuation verhinderte. Oberfläche glatt. Compression des Tumors vergeblich. Drei Tage nach Geburtsbeginn wurde die Sectio caesarea vorgenommen. Tod $40 \mathrm{~h}$. per operationem. Bei der Section fand man fünf Hydatidentumoren, die zusammen 15 Pfund wogen, davon vier in der Bauch-, eine in der Beckenhöhle. Von den in der Bauchhöhle gelegenen war einer in der hypogastrischen Gegend, zwei an der Stelle, die gewöhnlich Ovarien und Tuben einnehmen, einer neben dem Uterus. Im Becken aber war der Tumor, der die Geburt aufhielt, die Beckenhöhle überragend, mit Uteruskörper, Uterushals, Vagina und Rectum stark verwachsen, so dass man ihn, ohne diese Theile zu verletzen, nicht emporziehen konnte. Die vier Tumoren des Abdomen waren von Peritoneum, jener im Becken von Rectum und Vagina bedeckt. (Erzählt ist der Fall im Museum der Heilkunde der helvetischen Gesellschaft der Aerzte und Wundärzte B. II, XV, S. 211, von Dr. Meyer.)

5) Birnbaum.

42 jährige Frau, Mutter von zwei Kindern, deren jüngstes 12 Jahre alt. Seit mehreren Jahren Kränkeln, Kreuzschmerzen u. s. w., zuletzt Ischias dextra. Seit mehreren Jahren oft bei weitem Gehen 
plötzlich starker Harndrang, der zur Entleerung nöthigte. Menses im vergangenen" Jahre ausgeblieben.

Am 16. Juli Uterus handbreit über Symphyse, links neben ihm eine 3 " lange, $21 / 2 "$ breite, $2 "$ hohe knotige Geschwulst, schräg von der Synostosis puboiliaca aufsteigend; rechts neben dem Uterns, tiefer gegen die Beckenhöhle, eine zweite, rundlich breite, gleichmässige, deutlich vom Uterus abgegrenzt. Innerlich zeigte sich die ganze Kreuzbeinaushöhlung und der grösste Theil des Beckenraumes durch eine fast kindskopfgrosse, pralle, derbe Geschwulst ausgefüllt, die Portio vaginalis mit klaffenden Muttermundslippen über der Symphyse nach vorn - Retroflexio. Uterus spitz nach links emporsteigend, sich von der breiten Ausbiegung durch eine tiefe Bucht abgrenzend. Zwischen dieser Ausbiegung und dem Uterus fühlt man unter dem Nabel eine runde, glatte, verschiebbare Geschwulst. Anfang August erste Kindesbewegungeh, Anstossen eines spitzen Kindestheiles u. s. w. Im September Uterus mehr nach der Mitte gelegen, Befinden gut. Mitte October Uterus frei nach vorn, mitten und oben. Schieflage. Zeitweise geht blutiges Serum ab. 28. November, frïh $7 \mathrm{Uhr}$ plötzlich ohne Wehen viel Fruehtwasser entleert. Die Geschwulst fullte das Becken stark aus, so dass nur mit Mühe zwei Finger an den, über dem vorderen Beckenrande stehenden Mattermund gebracht werden konnten. Zeitweise Wasserabgang. Abends 7 Uhr Muttermund etwas leichter erreichbar, viex Querfinger breit. Abends $10 \mathrm{Ubr}$ vergeblicher Versuch, die Geschwulst zu reponiren. 29. November gegen Morgen heftige Drangwehen. Kopf des Kindes oben tief in die Geschwulst, die unten in starker Spannung vor ihm hergequollen ist, eingedrïckt. Muttermund quer verzogen, weit nach vorn unten von der Geschwulst ïberragt. Diese, nicht reponirbar, bot einzelne prall eindrückbare Stellen. Von 6-12 Uhr keine Verändernng trotz stürmischer Wehen. Deshalb mit Flourent'schem Troicar ein 2" tiefer Einstich in die Geschwulst. Etwas schwarzes Blut und wässerig-blutige Flüssigkeit; zweiter Einstich an einer anderen Stelle. Nach 1 h. starke Wehen mit krampf haft nervösen Reizsymptomen. Geschwulst hinter den Kopf zurückgezogen. Dieser im Beckeneingange. Muttermund fast verstrichen. In Narkose mit Forceps ein 51/2 Pfund schwerer, schlecht genährter Knabe entwickelt, der gleich darauf stirbt. Placenta durch Fingehen entfernt. Uterus leer.

Die ersten fünf Tage des Wochenbettes normal, dann Fieber u. s. w. Urin unter heftigen Schmerzen und Drang bald spärlich, bald in grosser Menge, trübe, aashaft stinkend. Geschwulst dxängte im Becken den Uterus sammt Uterushals immer noch entschieden nach links und vorn, wurde schmerzhaft, die kleine, bewegliche Geschwulst und der geschwulstartige Strang rechts waren noch zu fühlen, wie auch unter der Leber tief unter den Bauchdecken eine apfelgrosse Geschwulst. 12. December nach plötzlichem Abgange einer grossen Wassermenge heftiger Schüttelfrost, gefolgt von allgemeinen Krämpfen hysterischer Natur. 15. December yöllige Harnverhaltung, worauf mit wehenartigen Schmerzen durch die Urethra eine grosse, in ein $2-3^{2}$ langes, $1 / 2 "$ 
dickes Paket zusammengerollte Haut abging, der $6-8$ taubeneigrosse Blasen folgten. In den nächsten acht Tagen noch öfter Abgang solcher Blasen durch die Urethra, auch durch den angelegten Katheter.

Später Wohlbefinden. Uterus normal fungirend. Rudimente der Geschwulst zu fühlen: 1) unter der Leber, 2) in der Nabelgegend, 3) rechts von der Synostosis pubo-iliaca längs der Linea arcuata zur Synostosis sacro-iliaca strangartig sich binziehend.

6) Sadier.

21jährige I para, im neunten Monate schwanger. Schon seit der Kindheit merkliche Ausdehnung des Leibes.

Am 19. April Abfluss von Fruchtwasser, am 26. erste Untersuchung. Heftige Wehen. Finger nur mit Muhe zur Portio vaginalis zu bringen, da vom Promontorium her eine harte Geschwulst nach der Symphysis pubis sich erstreckte. Portio vaginalis war fest und nicht verstrichen. Kopf des Kindes durch den Muttermund noch zu erreichen. Da das Allgemeinbefinden befriedigend, beschloss man, bis zum anderen Tage zu warten, jetzt aber trat Verfall der Kräfte ein, so dass wegen der Diagnose einer festen Beckengeschwulst und eines kleinsten Beckendurchmessers von nur $11 / 4$ "der Kaiserschnitt beschlossen und ausgeführt wurde. Todtes, reifes Kind. Von der leeren Uterushöhle aus überzeugte man sich nochmals von der Verengerung des Beckens. Frau starb folgenden Tag.

Die Section ergab eine grosse Hydatidencyste, welche den ganzen oberen Raum der Lebergegend einnahm und bis ins Becken hinab hinter den Uterus sich erstreckte. Durch den anhaltenden Druck des Uterus hatte das härtige Gebilde täuschend die Härte eines Knochens simulirt. Nr. 184.)

7) Fall aus unserer Poliklinik. (Poliklinisches Journal 1875/76

L. G., Ipara, 26 Jahre. Inmer gesund gewesen von Seite des Abdomen keine besonderen Beschwerden. Von der Anwesenheit der Beckentumoren hat Pat. gelegentlich einer früheren Untersuchung durch einen Arzt Kenntniss erhalten.

Wehenbeginn 6. August früh 8 Uhr. Abends 6 Uhr erste Untersuchung. Mittelgrosse Figur, keine Zeichen von Rachitis. Wehen fehlen, kindliche Herztöne gut. Man bemerkt über der Symphyse etwas nach rechts von der Mittellinie einen fast doppelfaustgrossen Tumor. Er fühlt sich prall und elastisch an, lässt sich etwas verschieben und hängt durch ein kurzes, gerade nach hinten gehendes Ligament mit dem Uterus zusammen. Desgleichen geht vom Tumor ein anderes, deutlich zu fühlendes Ligament nach rechts hinten. Keine Fluctuation. Der ganze Uterus stark rechts liegend, Rücken des Kindes links. Der Douglas'sche Raum ausgefiullt mit zwei von links nach rechts berüberziehenden, elastischen Tumoren, von denen der linke faust-, der rechte apfelgross ist. Dieselben machen, ohne besonders resistent zu sein, den Eindruck solider Geschwïlste. . Sie sind anscheinend locker zusammenhängend und erstrecken sich so weit nach oben, dass 
dadurch das Promontorium verdeckt wird. Die Zurückschiebung der Tumoren in das Becken gelingt weder von der Scheide, noch vom Mastdarme aus. Portio vaginalis über den Beckeneingang nach vorn gedrängt, für zwei Finger durchgängig. Blase schlaff gespannt, Kopf beweglich, Sutura sagittalis querverlaufend, grosse Fontanelle etwa in der Mitte. Die Tumoren verengern den Beckeneingang derart, dass kaum drei Querfinger zwischen dieselben und die Symphyse gebracht werden können.

Da vorderhand ein Eingriff nicht nöthig, auch nicht möglich ist, wird abgewartet.

Abends $10 \mathrm{Uhr}$. Seit kurzer Zeit bessere Wehen. Kopfstellung und Grösse ${ }^{\bullet}$ des Muttermundes wie zuvor.

Ich liess in Voraussicht einer sehr schwierigen Entbindung die Kreissende in die Klinik transferiren. Hier wurde nach vorheriger Consultation mit dem von mir, in Abwesenheit des Herrn Professor Spiegelberg, zugezogenen Director der Hebammenschule, Herrn Dr. Langer, Nachts $12 \mathrm{Uhr}$ in Chloroformnarkose noch einmal eine genaue Untersuchung vorgenommen und mit der ganzen Hand vom Rectum aus abermals die Zurïckschiebung der Tumoren versucht, allein vergeblich. Man konnte dabei leicht constatiren, dass sich der linke Tumor bis iiber das Promontorium hinauf erstreckte und dasselbe ganz verdeckte. Es wurde vom Rectum aus der linke Tumor punctirt, und es flossen etwa 200 Gramme einer trüben, gelblichen Flüssigkeit ab. Dann wurde von der Scheide aus der zugänglichere, rechts liegende kleinere Tumor punctirt, und auch hier floss dieselbe Flüssigkeit - jedoch nicht so viel - aus. Alsbald waren beide Tumoren für die Palpation verschwunden. Die Blase war unabsichtlich gesprengt worden und stark mit Meconium vermischtes Fruchtwasser abgegangen. Der Kopf hatte sich nach der Punction ziemlich fest auf den Beckeneingang gestemmt, Sutura sagittalis quer in der Mitte zwisehen Symphyse und Promontorium, grosse Fontanelle tiefer stehend.

Die Beckenmaasse waren:

Spinae ilei 22,0 c.

Cristae ilei 23,5 c.

Conjugata externa e. 17,75 c.

Conjugata diagonalis etwa $11,5 \mathrm{c}$.

Die rechte Spina ischii springt etwas stark hervor.

$\mathrm{Da}$ die kindlichen Herztöne, sowie das Befinden der Kreissenden gut, die Beckenverengerung nur gering, desgleichen der über der Symphyse befindliche grosse Tumor die Geburt nicht zu hindern schien, wurde der weitere Verlauf abgewartet.

7. August früh 6 Uhr. Starke Wehen. Kopf fast ganz durch den Beckeneingang getreten, nahe dem Beckenboden. Grosse Fontanelle tiefstehend. Muttermund vollștändig eröffnet. Oedem der Vulva, blutiger Harn. Kindliche Herztöne nicht mehr za hören. Unter diesen Umständen Perforation mit Scheere durch die grosse Fonta- 
nelle und Anlegung des Kranioklast. Der Kopf folgt diesem nicht, die Knochen geben nach, ebenso bei wiederholtem Anlegen an anderen Stellen. Endlich lege ich den Kranioklast noch einmal nach hinten über die linke Seite des Gesichtes, und jetzt folgt der Kopf bei starkem Zuge. Durch festes Ziehen am Halse wird auch der Rumpf entwickelt; ihm folgt sofort die Placenta. Ziemliche Blutung. Bei der Untersuching zeigt sich ein Riss in der linken Seite der Cervix. Wöchnerin klagt, aus der Narkose erwacht, über starke Schmerzen im Leibe. Sie stirbt unter den Zeichen acuter Peritonitis am nächsten Tage Vormittags $91 / 2$ Uhr.

Die (durch Herrn Dr. Weigert vorgenommene) Section ergab: Reichliche Verwachsung des Uterus mit seiner Umgebung, mit der vorderen Bauchwand, mit dem Netze. Zahlreiche, bis kindskopfgrosse Echinococcen vor, hinter und seitlich vom Uterus. Echinococcus im grossen Netze, ein abgestorbener in der Leber. Zerreissung der linken Seitenwand der Cervix uteri vom inneren Muttermunde bis zwei Finger vom äusseren entfernt, Suffusion des parametranen Bindegewebes und Zerreissung des Peritoneum an derselben Seite. Chronische und acute Peritonitis.

Die sieben Beobachtungen haben, wie man sieht, verschiedene gemeinsame Eigenthümlichkeiten.

So liegen fast sämmtliche Säcke zwischen Vagina und Rectum, entweder gerade nach hinten oder seitlich im kleinen Becken. Das lockere subseröse Zellengewebe - wie schon Davaine bemerkt, sitzen die Beckenhydatiden gewöhnlich hier - seitlich vom Uterus bietet natürlich der Entwickelung der Echinococcentumoren einen günstigen Boden. Auffallend aber ist, dass die Mehrzahl der Kysten gerade nach hinten im retrocervicalen und -vaginalen Bindegewebe lag, während in dem zwischen Blase und Uterus befindlichen kein Echinococcus beobachtet wurde. Uebrigens kommen auch im Douglasschen Raume Hydatiden vor, die nach Davaine ursprünglich im Ovarium entstanden, dann in den Douglas'schen Raum gefallen und dort weiter gewachsen sind. (Siehe Davaine, Obs. 150, 151.)

Ferner zeichneten sich die Beckenechinococcen durch ausserordentliche Härte aus. Diese Resistenz ist bedingt durch den bei Gebärenden beträchtlich verstärkten Druck, dem entsprechend natürlich auch der Druck des Kysteninhaltes auf die Kystenwand erhöht wird. Die Folge davon ist stärkere Spannung der Wand, die beim Untersuchen den Eindruck eines festen, soliden Tumors hervorruft. Dass dieses Gefühl der Härte da, wo der kindliche Kopf durch die Wehen bereits fest auf den Tumor aufgedrängt ist, noch verstärkt sein muss, ist einleuchtend. Der gesteigerte Druck, unter dem der Kysteninhalt steht, ist auch die Ursache davon, dass bei Beckenechinococcen fast nie Fluctuation oder Hydatidenschwirren geftihlt wurde. In sämmtlichen bei $D$ avaine angeführten Fällen war nur in einem Falle (Obs. 167) dieses Schwirren gefühlt worden. 
Weiter finden wir, dass in keinem Falle eine Reposition der Tumoren in die Abdominalhöhle möglich war. Dies ist die Folge der subserösen Lage und der zahlreichen Adhäsionen, welche unter dem Reize des Parasiten gebildet wurden.

Die Oberfläche der Kysten ist meist glatt. Jedoch ist dies nicht immer der Fall, da grössere Tochterkysten der Haupt- (Mutter-) Kyste eine höckerige 13eschaffenheit verleihen können.

Die Hauptbeschwerden betrafen, wie es auch der tiefen Lage der Echinococcusgeschwïlste im kleinen Becken entspricht, vorzüglich die Harn- und Stuhlentleerung. Anderweitige Störungen, wie Kreuzschmerzen, Zerrung im Becken u. s. w. kamen seltener vor (Roux, Birnbaum). Auch in den übrigen Fällen von Beckenhydatiden bei Davaine sind fast nur Störungen der Blase nnd des Darmes beobachtet worden. (Bei einigen wurde zufällig in der Leiche Erweiterung der Ureteren, Nierenbecken u. s. w. gefunden. $\mathrm{Ob}$ besondere Störungen von Seite der Nieren vorhanden waren, lässt sich nicht ersehen.) Merkwürdigerweise war fast in allen Fällen die Menstruation entweder vollkommen normal, oder nur ganz gering gestört. $\mathrm{Ja}$ in unserem Falle hatte die Kranke gar keine Beschwerden von Seite der sehr umfangreichen Tumoren, so dass sie erst gelegentlich einer ärztlichen Untersuchung, wie schon erwähnt, Kenntniss von ihren Beckengeschwülsten erhielt.

In drei von diesen sieben Fällen waren zugleich Kysten anderer Abdominalorgane vorhanden ( $\mathrm{Pu}$ ehelt, Birnbaum, unserer). Unter Umständen könnte auf Grund der Thatsache, dass man neben Beckentumoren zugleich Tumoren anderer Körperregionen, die erfahrungsgemäss Lieblingssitz der Echionococcen sind, z. B. der Lebergegend, findet, die Eruirung der wirklichen Beschaffenheit solcher Geschwülste ermöglicht werden. (Von 27 Fällen bei Davaine waren $17 \mathrm{Mal}$ mehrere Kysten vorhanden.)

Mit Ausnahme des Roux'schen Falles, der ja überhaupt zweifelhaft ist, hatten die Tumoren eine derartige Grösse erreicht, dass sie durch Verlegung des Beckeneinganges ein absolutes Geburtshinderniss abgaben. In allen diesen Fällen hatten sie, wie alle retrovaginalen und retrocervicalen Geschwülste, den unteren Uterinabschnitt nach oben und vorn gedrängt, so dass der Scheidentheil nur mit 2-3 Fingern hoch über der Symphyse erreicht werden konnte. Ein Eingehen mit ganzer Hand war in keinem Falle möglich. Immer musste operative Kunsthülfe eintreten, da die heftigsten, zum Theil langdauernden Wehen den Eintritt des Kopfes nicht erzielten. Nur bei der Patientin Park's verlief die erste Geburt nach langer Anstrengung, und eine weitere im siebenten Monate spontan. Bei einer späteren Geburt am normalen Ende musste jedoch auch die Verkleinerung des Tumors vorgenommen werden.

Das eingeschlagene Operationsverfahren bestand bei Park, Blot, Birnba um und in unserem Falle in Punction resp. Incision der Tumoren. In den Fällen von Puchelt und Sadler wurde im Glauben an die solide Beschaffenheit der Tumoren die Sectio caesarea 
vorgenommen. Bemerkenswerth ist, dass ziemlich bald nach Vornahme der Punction in allen Füllen der Kopf tiefer trat.

Die Diagnose wird wohl in der Regel nur durch Ausschliessung zu stellen sein, da die positiven Zeichen (Hydatidenschwirren) selten wahrzunehmen sind. So giebt Davaine an, dass Verwechselung einer Hydatidenkyste mit einem Beckenabseess nicht leicht sei. Die Richtigkeit dieser Aussage lässt sich nicht bestreiten, da Beckenabscesse meist nicht chronischen Charakters und Folge anderweitiger Leiden sind.

Eine Verwechselung mit Blutgeschwülsten (Haematokele) ist aus dem Grunde nicht leicht möglich, weil letztere in der Gravidität bis jetzt nicht beobachtet sind. Von fibrösen und carcinomatösen Tumoren werden Hydatiden nach Davaine's Meinung leicht durch ihre Consistenz unterschieden. Es wird - dies jedoch sehr oft nicht möglich sein, da gerade Beckenechinococcen, wie wir oben sahen, oft die Härte solider Tumoren darbieten.

Die Erkennung der Art der Kyste kann nur die Punction ermöglichen.

Spencer Wells sagt hierzu (Diseases of the ovaries, pag. 151): Bei Hydatiden beginnt die Vergrösserung gewöhnlich im oberen Theile des Abdomen und geht dann erst aufs Becken über. Dieselbe Ansicht äussert auch Beigel in seinen „Krankheiten des weiblichen Geschlechtes" S. 503. Doch ist dies nur eine Angabe, über deren Richtigkeit wir kein positives Urtheil haben, da die Hülfe meist erst requirirt wird, wenn die Tumoren bereits tiefer in das Becken herabgetreten sind. Wenig stichhaltig sind die übrigen von Spencer Wells angegebenen Unterscheidungsmerkmale, nämlich dass Hyda. tiden in der Regel schneller wachsen als Ovarialkysten, dass sie unregelmässige Oberfläche haben können, dass die Fluctuation bei ihnen sehr unscheinbar und dunkel, und dass der tympanitische Schall meist seitlich ist. Alle diese Merkmale können auch bei Ovarialkysten vorhanden sein. Die Unterscheidung von Ovarialtumoren kann also recht schwierig sein, zumal bei letzteren sowohl Menstruationsstörungen, als auch andere bedeutendere Beschwerden gänzlich fehlen können.

Von parametrischen Exsudaten, die doch fast immer erhebliche Beschwerden mit sich bringen und erhöhtes Krankheitsgefühl erzeugen, wird man die Hydatiden durch die Anamnese und meist wohl auch durch den localen Befund,' von Exostosen der Beckenknochen aber einerseits durch das Gefühl und andererseits dadurch unterscheiden können, dass Exostosen unbeweglich, Hydatiden aber in gewissem Grade beweglich und verschiebbar sind.

Nach dem bisher Gesagten müssen wir uns also dahin aussprechen, dass wir. einen Echinococcus dann vermuthen dürfen; wenn man in der Beckenhöhle einen oder mehrere glatte, pralle, etwas verschiebbare, dem Uterus nicht angehörende Geschwülste, die sich ohne grosse Beschwerden entwickelt haben, findet, wenn dabei die geschlechtlichen Functionen wenig oder gar nicht gestört sind, wenn ferner das Allgemeinbefinden nicht einer malignen Erkrankung ent- 
spricht, und wenn sich endlich noch an anderen Stellen ähnliche Tumoren vorfinden. Zur Gewissheit wird die Vermuthung, wenn es gelingt, deutliches Hydatidenschwirren zu fühlen.

Die Punction natürlich entscheidet. Sie bahnt auch den Weg zur richtigen Behandlung. Man soll sie daher bei zweifelhaften Beckentumoren unter allen Umständen vornehmen. Jedenfalls darf man sich durch Härte und Resistenz des Tumors nicht verleiten lassen, sie zu 'unterlassen und, wie im Puchelt- und Sadler'schen Falle, zum gefährlichsten Entbindungsmittel, zur Sectio caesarea zu greifen. Sadler bemerkt in Betreff seines Falles selbst, dass man durch Erkenntniss der wahren Natur des Tumors bei der Lebenden durch einen Einschnitt oder Stich das Geburtshinderniss hätte wahrscheinlich leicht beseitigen können.

Ist die Probepunction erfolgreich, so entfernt sie sofort das Geburtshinderniss. Man warte aber nicht allzu lange, da bei heftigen Wehen entweder eine Ruptura uteri oder bei kystischen Tumoren unter dem andrängenden Kopfe eine Ruptur derselben in die Abdominalhöhle erfolgen kann. Man verlasse sich auch nicht darauf, dass vielleicht mit dem Tieferdrücken des Kopfes eine vorher nicht zu reponirende Geschwulst sich allmälig über den Beckeneingang zurückziehen könne. Es ist ja doch sehr zweifelhaft, ob diese Eventualität eintreten wird, und wenn sie auch eintritt, sind nach unserer Ansicht nicht alle Gefahren, die vom Tumor drohen, damit beseitigt. Wir glauben nämlich die in unserem Falle gefundene Cervical- und Peritonealruptur dadurch erklären zu müssen, dass einer oder mehrere der zahlreichen, den Uterus umgebenden, mit demselben innig verwachsenen und von der Punction nicht getroffenen Echinococcustumoren beim Tiefertreten des Kopfes und unter den Uteruscontractionen zwischen kindlichem Halse oder schultern und Beekenwand hereingepresst wurden und so das Vorrücken des Kindes hinderten. Durch die gespannten äusseren Bauchdecken hindurch war dieser Vorgang allerdings nicht zu beobachten. Allein wie könnte man sich sonst die Thatsache erklären, dass trotz heftiger Wehen der Kopf in der Beckenhöhle nicht vorriickte und erst nach langem, starkem Zuge mit dem Kranioklast entwickelt werden konnte? Vermuthlich wirkte der Zug des Kranioklast weniger auf den kindlichen Rumpf, als auf den gleichzeitig mit herabgezogenen "Uterus und Tumor, so dass bedeutende Zerrung und Dehnung und endlich Zerreissung der Cervicalwand erfolgte. Deshalb wurde auch schon bemerkt, dass mit der Reposition eines Tumors nicht sofort alle Gefahren beseitigt sind.

Dass die Punction in den hier zusammengestellten Beobachtungen recht zufriedenstellende Resultate erzielte, geht schon daraus hervor, dass die Geburt bei Allen bald darauf beendet wurde, und dass, mit Ausnabme des unserigen, alle Fälle einen günstigen Ausgang nahmen. Und in unserem Falle lag, wie wir oben sahen, die Ursache des lethalen Ausganges nicht in der Punction, sondern in anderen Verhältnissen. Punetionen von der Vagina aus scheinen überhaupt weniger gefährlich zu sein, als die von der Bauchwand 
her, da die Beckenserosa allem Anscheine nach weniger heftig reagirt, als die Bauchserosa. Allerdings trat fast immer langdauernde Eiterung im Hydatidensacke ein. Allein auch diese wird man durch die heutigen vervollkommneten antiseptischen Behandlungsmethoden abkïrzen und vermindern können. Es sei auch bemerkt, dass die Punction per vaginam der per rectum vorzuziehen ist, weil bei letzterer die Gefalır des Gaseindringens ùnd somit der Jauchung viel grösser ist, als bei ersterer. Auch aus den Zusammenstellungen Davaine's sieht man, dass die Punction per vaginam die günstigsten Resultate der Behandlung von Beckenhydatiden liefert.

In allen dort erwähnten Fällen trat nur fünf Mal Heilung ein, darunter die drei hier erzählten Beobachtungen mit Punction resp. Incision von der Scheide aus. (Die zwei anderen Heilungen betreffen Männer. Das eine Mal wurde vom Perineum aus incidirt, das andere Mal erfolgte spontaner Durchbruch der Hydatidenmassen durch den Anus.) Alle durch die Bauchwand Punctirten, ebenso eine von der Vagina und eine vom Rectum aus Punctirte starben. Die übrigen gingen an Marasmus zu Grunde (bei einem Theile wurden die Hydatiden zufällig in der Leiche gefunden). Spontane Oeffnung in die Nachbarorgane kam fünf Mal vor. Davaine bemerkt hierzu: „Man sieht nach diesen Thatsachen, dass die Hydatiden, welche das Becken zum Sitze haben, unter die schwersten gezählt werden dürfen."

Der Vollständigkeit halber seien hier noch einige andere Beobachtungen, welche nicht an Puerperen gemacht wurden, mitgetheilt:

Schatz (dieses Archiv IX. Bd. S. 115).

1) Echinococcus im Ligamentum latum. Punction rechts von der Portio vaginalis. Tod nach 14 Tagen an Peritonitis.

2) Echinococcus der Fossa iliaca sinistra. Punetion, Heilung.

Scherenberg (Virchow's Archiv, Bd. 46, S. 392), Enormer Echinococcus des Netzes. Verwechselung mit Hydrops ovarii. Punction. Tod nach sechs Wochen an Inanition.

Newman (Transaction of the obstetrical society of London, Vol. IV, 1862).

Echinococcus der Leber, des Netzes, des Beckens, um den Uterus, Blase, Ovarium gelagert $u$. s. w. Incision zwischen Wirbelsäule und Crista ossis ilium dextri. Heilung. Zwei Jahre später Tod an Peritonitis.

Cailleux (Gazette des hôpitaux 1868, p. 366).

Hydatidencyste des Musculus iliopsoas. Incision an der Innenfläche des linken Schenkels. Heilung.

Freund und Chadwick (The American journal of obstetrics, IV, p. 668).

1) Echinococcus in der hinteren Wand des Uterus. Punction nahe der Portio vaginalis. Vergrösserung der Oeffnung mit Bistouri. 
Nene Colonien im Beckenzellgewebe. Spontane Perforation in Blase und Rectum. Heilung.

2) Echinococeus in Leber, Milz, grossem Netze und Beckenzellgewebe. Exquisites Hydatidenschwirren. Punction durch die Scheide. Heilung.

3) Echinococcus in Leber, Milz, grossem Netze und vielleicht auch im rechten Ovarium (?) Hydatidenschwirren. Drei Mal Punction des linken Leberlappens, ein Mal Punction des Netzes. Heilung. Gestorben.

4) Echinococcus hepatischen Ursprungs im Do u g la s'schen Raume.

Beigel (Krankheiten des weiblichen Geschlechts, S. 503).

Echinococcus des Omentum majus, drei Echinococcusblasen in der Uterinhöhle. Punction vom Abdomen aus. Zwei Jahre später Tod an Peritonitis.

Graily Hewitt (Transactions of the obstetrical society of London, Vol. XII, p. 135).

Hydatiden des Uterus. Injection von Liquor ferri. Heilung.

Jedenfalls geht aus diesen Beobachtungen hervor, dass die Punction der Beckenhydatiden in den Fällen wenig gefahrdrohend ist, in denen eine Verletzung des Peritoneum vermieden werden kann.

Schliesslich will ich noch auf die interessante Beobachtung Park's aufmerksam machen, dass durch Vernarbung und Schrumpfung punctirter Beckenhydatiden der Genitalschlauch derart verengt werden kann, dass bei einer späteren Geburt ein ernstliches Geburtshinderniss daraus entsteht.

\title{
Mittheilungen aus der Gesellschaft für Geburtshülfe in Leipzig.
}

\author{
Sitzung am 15. Januar 1877.
}

Herr Ahlfeld berichtet über einen Fall von doppelten äusseren Geschlechtstheilen bei einem sechs Monate alten Mädchen.

Dieser von Dr. Suppiger in Triengen (Ct. Luzern) beschriebene Fall (Schweizer Correspondenzblatt, 1876, Nr. 14) ist als ein Unicum von hohem Interesse. Ahlfeld hat durch die Gite des Dr. Suppiger eine Zeichnung der verbildeten Genitalien erhalten, 